\title{
Probing Short-Range Correlations in Nuclei with Neutrinos, Protons, and Electrons
}

\author{
Stephen A. Wood \\ Thomas Jefferson National Accelerator Facility
}

\begin{abstract}
Experimental signatures for short-range correlations using electron, proton and neutrino probes are discussed. The measurement of ${ }^{12} \mathrm{C}\left(e, e^{\prime} p p\right)$ and ${ }^{12} \mathrm{C}\left(e, e^{\prime} p n\right)$ indicates that correlations of $n p$ pairs dominate that of $p p$ pairs.
\end{abstract}

Keywords: Short Range Correlations

PACS: 21.30.Fe, 21.60.-n, 24.10.-i, 25.30.-c

\section{INTRODUCTION}

The Independent Particle Model (IPM) and Shell Model of nuclei are quite successful. However, it has long been believed that the strong nucleon-nucleon force and it's repulsive short range behavior should give rise to observable short-range correlations (SRC) of nucleons in nuclei. The case for the observation of SRC has only been built slowly, in part because of difficulty of the experiments and the need to disentangle SRC from other nuclear effects.

One manifestation of SRC is the emission of backward protons (relative to the beam direction) in neutrino and electron scattering on nuclei. In the absence of nuclear effects such as correlations and final-state interactions, such backward protons are kinematically forbidden. Neutrino induced backward protons in the NOMAD detector were discussed in the context of SRC at NUINT01 [1]. This was of interest to the author as an experiment to study $\mathrm{SRC}$ with the triple coincidence reaction ${ }^{12} \mathrm{C}\left(e, e^{\prime} p N\right)$, where one of the nucleons is backward going, had been recently approved at the Thomas Jefferson National Accelerator Facility (JLab) [2].

\section{EVIDENCE FOR SHORT RANGE CORRELATIONS}

The distribution of nucleons within a nucleus can be described by a spectral function, $S(\mathbf{p}, E)$, that gives the probability of finding a nucleon with momentum $\mathbf{p}$ that leaves the nucleus with an excitation of $E$ when removed from the nucleus. In independent particle models, the strength of the spectral function is limited to momenta and energies less than $250 \mathrm{MeV} / c$ and $30 \mathrm{MeV}$, namely the Fermi momentum and energy. In models built up from realistic nucleon-nucleon potentials, which include a repulsive core and a medium range tensor component, the spectral function strength in the Fermi-gas region is reduced by about $20 \%[3,4]$. Measurements with reactions such as $\left(e, e^{\prime} p\right)$ show a reduction of at least 30\% [5], which is consistent with the expectation that short-range correlations are an important part of the description of the nucleus. Actually observing spectral function 


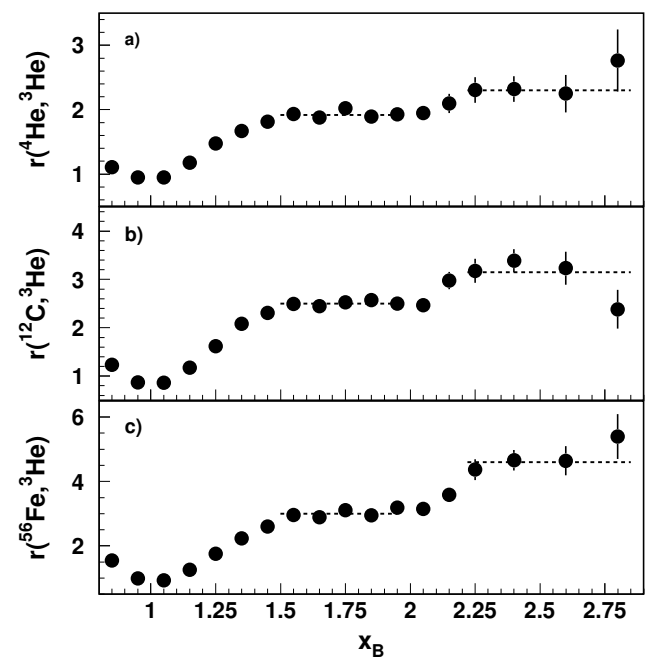

FIGURE 1. Weighted $\left(e, e^{\prime}\right)$ cross section ratios, from Egiyan et al.[9], of (a) ${ }^{4} \mathrm{He}$, (b) ${ }^{12} \mathrm{C}$ and (c) ${ }^{56} \mathrm{Fe}$ to ${ }^{3} \mathrm{He}$ as a function of $x_{B}$ for $Q^{2}>1.4(\mathrm{GeV} / c)^{2}$. The horizontal dashed lines indicate the $2 N$ and $3 N$ SRC scaling regions.

strength at higher $\mathbf{p}$ and $E$ has been difficult because this strength is spread over a large momentum and energy range. Recently, strength at high $(\mathbf{p}, E)$ has been observed [6] and is consistent with predictions that include short-range correlations.

Inclusive electron scattering, $A\left(e, e^{\prime}\right)$, can be used to probe SRC. In inclusive electron scattering, the kinematics of the scattered electron are characterized by $x_{B}=Q^{2} /(2 M v)$, where $M$ is the nucleon mass and $v$ is the energy transferred to the nucleus. $x_{B}$ can not be greater than one unless the electron scatters off of a moving nucleon or an object with a mass greater than the mass of a nucleon. It has been observed that ratio of inclusive electron scattering between heavy and light nuclei scales (is independent of $x_{B}$ and $Q^{2}$ ) for $x_{B}$ somewhat above one [7,8]. This scaling has also been observed at $x_{B}$ above two, probing 3 body correlations. (Fig. 1) This scaling, which was predicted by SRC models, indicates that the shapes of nucleon momentum distributions for momenta above the Fermi momentum are similar for both light and heavy nuclei. Using the scaling ratios and ${ }^{3} \mathrm{He}$ wavefunctions, the per-nucleon probability to be in a short range correlations can be calculated [9]. For carbon, this probability is about $20 \%$ for two nucleon correlations and about $0.5 \%$ for three nucleon correlations.

\section{TRIPLE COINCIDENCE EXPERIMENTS}

In the above experiments, it is assumed that high momentum nucleons in a nucleus are due to SRC and that observation of high momentum components is a signature of SRC. While is assumption is reasonable, additional information about SRC can be gained by observing both nucleons in a correlated pair. If nucleon in a correlated pair is knocked out of a nucleus, the "paired" nucleon should be emitted in the opposite direction of the 
initial momentum of the knocked out nucleon. Detecting both nucleons can address such questions as 1) how often is a high momentum nucleon paired with a single opposite momentum nucleon, 2) what is the center of mass motion of correlated pairs, and 3) are $n p$ or $p p$ (and $n n$ ) SRC pairs more likely.

At Brookhaven, the EVA spectrometer and neutron detectors were used to measure the triple coincidence reaction ${ }^{12} \mathrm{C}(p, 2 p n)$ with beam momenta between 5.9 and $9.0 \mathrm{GeV} / c$ [10]. If it is assumed that two outgoing protons are the result of a quasi-elastic scattering between the beam and a bound proton, then the initial momentum of the bound proton is $\mathbf{p}_{i}=\mathbf{p}_{1}+\mathbf{p}_{2}-\mathbf{p}_{b}$, where $\mathbf{p}_{b}$ is the beam momentum and $\mathbf{p}_{1,2}$ are the momenta of the detected protons. With cuts chosen to emphasize quasi-elastic scattering, the correlation of the neutron direction with respect to $\mathbf{p}_{i}$ was examined. For neutron momenta below $220 \mathrm{MeV} / c$, no correlation was found, while above $220 \mathrm{MeV} / c$, the neutron direction was peaked backward from $\mathbf{p}_{i}$ with almost all events having a relative angle greater than $90^{\circ}$. These results are strongly suggestive of a neutron-proton pair, with high relative momentum, being broken apart by the proton beam.

A similar experiment, simultaneous measurement of ${ }^{12} \mathrm{C}\left(e, e^{\prime} p p\right)$ and ${ }^{12} \mathrm{C}\left(e, e^{\prime} p n\right)$, was recently carried out at JLab [2]. The use of the electron beam and the choice of kinematics with a high $Q^{2}$ of $2(\mathrm{GeV} / c)^{2}$ and $x_{b}=1.2$ favors the picture of the electron interacting with and knocking a single high momentum nucleon out of the nucleus. The kinematics of the first proton were chosen such that $\mathbf{p}_{m}=\mathbf{p}_{e}+\mathbf{p}_{p}-\mathbf{p}_{b}$, the momentum of the proton before the interaction, had values ranging from $300-600 \mathrm{MeV} / c$. A proton spectrometer [11] and neutron array, each with a solid angle of $\approx 100 \mathrm{msr}$, were placed at an angle directly opposite of $\mathbf{p}_{m}$. After correcting for the limited solid angle, it was found that $(9.5 \pm 2) \%$ of ${ }^{12} \mathrm{C}\left(e, e^{\prime} p\right)$ at high $\mathbf{p}_{m}$ was accompanied by a "backward" proton [12], suggesting that short range correlated $p p$ pairs are infrequent. Furthermore, observation of neutrons, showed a ratio of ${ }^{12} \mathrm{C}\left(e, e^{\prime} p n\right)$ over ${ }^{12} \mathrm{C}\left(e, e^{\prime} p p\right)$ of $8 \pm 2$ [13]. As the ${ }^{12} \mathrm{C}\left(e, e^{\prime} n p\right)$ reactions were not measured, this indicates that $n p$ correlations are as much as 20 times more prevalent than $p p$ correlations. This preponderance of $n p$ correlations is also seen in a reanalysis of the Brookhaven data [14]. Calculations of the ground state structure of nuclei show that the short range part of the $N-N$ tensor force leads to a dominance of $n p$ to $p p$ pairs for high relative momenta [15].

\section{FUTURE EXPERIMENTS}

A measurement of ${ }^{4} \mathrm{He}\left(e, e^{\prime} p N\right)$ is planned at JLab [16]. The $n p$ to $p p$ ratio will be mapped out as a function of $\mathbf{p}_{m}$. The ratio is expected to have a stronger dependence on $\mathbf{p}_{m}$ for lighter nuclei.

A difficulty with making measurements with electron beams is the high accidental rate that competes with the detection of triple coincidence events. There is a potential that finely grained fully active neutrino detectors such as MINER $v$ A can add to the study of SRC through the ${ }^{12} \mathrm{C}\left(v, \mu^{-} p p\right)$ reaction which is sensitive $n p$ pairs. In a four year run with MINER $v A$, several thousand quasi-elastic $\mathrm{CC}$ events at relevant kinematics $\left(x_{B}>1, p_{m}>300 \mathrm{MeV} / c\right)$ will be recorded. This data set may be competitive with electron scattering in answering such questions as what fraction of the high momentum tail of the nucleon momentum distribution is due to SRC and what is the center of mass 
momentum distribution of correlated pairs.

\section{ACKNOWLEDGMENTS}

The author thanks the E01-015 collaboration. This work was supported by the U.S. Department of Energy; Jefferson Science Associates, LLC operates the Thomas Jefferson National Accelerator Facility under Department of Energy contract DE-AC05$06 \mathrm{OR} 23177$.

\section{REFERENCES}

1. M. Veltri, "A study of nuclear effects in $v$ interactions with the NOMAD detector," in Proceedings of NuInt01: The First International Workshop on Neutrino-Nucleus Interactions in the Few GeV Region, edited by J. G. Morfin, M. Sakuda, and Y. Suzuki, Nucl. Phys. B (Proc. Suppl.) 112, Elsevier Science B.V., The Netherlands, 2002.

2. E01-015: Studying the internal small-distance structure of nuclei via the triple coincidence $\left(e, e^{\prime} p+\right.$ n) measurement, Jefferson Lab Experiment Proposal (2001).

3. O. Benhar, A. Barocini, and S. Fantoni, Nucl. Phys. A 505 (1989).

4. O. Benhar, N. Farina, H. Nakamura, M. Sakuda, and Y. Seki, Phys. Rev. D 72 (2005).

5. L. Lapikás, Nucl. Phys. A $\mathbf{5 5 3}$ (1993).

6. D. Rohe, et al., Phys. Rev. Lett. 93 (2004).

7. D. B. Day, et al., Phys. Rev. Lett. 59 (1987).

8. K. S. Egiyan, et al., Phys. Rev. C 68 (2003).

9. $\quad$ K. S. Egiyan, et al., Phys. Rev. Lett. 96 (2006).

10. A. Tang, J. W. Watson, et al., Phys. Rev. Lett. 90, 042301 (2003).

11. R. Shneor, High Luminosity Operation of Large Solid Angle Scintillator Arrays in Jefferson Lab Hall A, Master's thesis, Tel Aviv Univeristy, Tel Aviv, Israel (2003).

12. R. Shneor, et al., Phys. Rev. Lett. (2007).

13. R. Subedi, Studying Short-Range Correlations with the ${ }^{12} \mathrm{C}\left(e, e^{\prime} p n\right)$ Reaction, Ph.D. thesis, Kent State University, Kent, OH 44242 (2007).

14. E. Piasetzky, M. Sargsian, L. Frankfurt, and J. W. Watson, Phys. Rev. Lett. 97, 162504 (2006).

15. R. Schiavilla, R. B. Wiringa, S. C. Pieper, and J. Carlson, Phys. Rev. Lett. 98 (2007).

16. E07-006: Studying short-range correlations in nuclei at the repulsive core limit via the triple coincidence $\left(e, e^{\prime} p n\right)$ reaction, Jefferson Lab Experiment Proposal (2007). 